\title{
Uncertainties confronting stakeholders and decision-makers in planning intervention in urban and agricultural scenarios in the transition phase of a radiological emergency
}

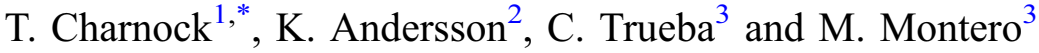 \\ ${ }^{1}$ PHE-Public Health England, Centre for Radiation, Chemical and Environmental Hazards, Didcot, UK. \\ ${ }^{2}$ DTU - Technical University of Denmark, Roskilde, Denmark. \\ ${ }^{3}$ CIEMAT - Centro de Investigaciones Energéticas, Medioambientales y Tecnológicas, Madrid, Spain.
}

\begin{abstract}
This paper summarises the work undertaken under task 1 of work package 4 of the CONFIDENCE project. The objective was to investigate uncertainties confronting stakeholders and decision makers at the transition phase of an accident and to frame the scene for subsequent tasks to investigate how stakeholders respond to that uncertainty. Urban and agricultural scenarios were considered separately. For urban scenarios, the investigation focussed on uncertainties in model predictions of residual dose from external exposure to radionuclides deposited in the urban environment. For agricultural scenarios, the objective was to analyse the uncertainties in the transfer and fate of radionuclides along different components of agricultural systems and their response to recovery actions.
\end{abstract}

Keywords: CONFIDENCE / uncertainties / urban / agriculture / transition / recovery

\section{Introduction}

The transition phase of a nuclear accident is the period in which decision-making pivots from protective actions for the immediate protection of human health to actions to promote and sustain human activity in the affected areas in the long term. It is characterised by increasing certainty about the current radiological situation; the state of the facility and the spread and characteristics of environmental radioactivity, while future residual doses and the contamination of agricultural systems, as well as the response of populations to these, remain uncertain.

The aim of WP4 was to improve the preparedness and response in the transition phase of an accident, by identifying and trying to reduce uncertainties in the subsequent planning of management of the long-term exposure situation. The accidental radioactive release can affect the environment and the human being through different routes of exposure. Generally, external exposure is the most significant pathway for urban scenarios, and the ingestion of contaminated foodstuffs is the most significant for agricultural scenarios. Both scenarios are complex and inhomogeneous, even within a particular region or area. Although elements of both scenarios will be experienced in real incidents, it is convenient to consider agricultural and urban scenarios separately, in order to

\footnotetext{
*Corresponding author: tom. charnock@phe.gov.uk
}

identify and quantify better the applicability of the possible recovery options.

Task 1 of WP4 included a review of urban and agricultural scenarios to identify and address sources of uncertainty. For urban scenarios, the focus was the uncertainties inherent in the prediction of residual doses since this underpins decision making. For agricultural scenarios, it was the behaviour and fate of radionuclides in the soil-plant system and their transfer along the food chain pathway. In WP4, the term "agricultural" was used very broadly to mean any area used with purposes of farming production, including grazing, where the products and the consumers are connected through the food-chain exposure pathway. No model to predict the residual dose was used, as in the case of the urban scenarios, instead, the objective was the assessment of the behaviour, fate and transfer of radionuclides along the different components that constitute the agricultural scenarios, their response to the recovery actions and the uncertainties identified in the overall process.

This paper summarises some of the findings that are reported in detail in Charnock et al. (2018) and in Montero et al. (2018).

\section{Urban scenarios}

Urban areas encompass diverse and interconnected domestic, social, commercial, economic and political activity, and so recovery presents distinct and on-going challenges to decision-makers because the disruption can be both large and 
Table 1. Deposition of caesium as a soluble aerosol onto different roof materials relative to the reference surface of lawn.

\begin{tabular}{|c|c|c|c|c|c|c|}
\hline Roof material & \multicolumn{2}{|c|}{ Wet rel. deposition } & \multicolumn{2}{|c|}{ Wet runoff fraction } & \multicolumn{2}{|c|}{ Dry rel. deposition } \\
\hline Current ERMIN defaults & 0.85 & 0.15 & 0.5 & 0.3 & 0.7 & 0.4 \\
\hline Clay tile & 0.8 & 0.2 & 0.3 & 0.04 & 0.8 & 0.1 \\
\hline Concrete tile & 0.8 & 0.2 & 0.4 & 0.05 & 1.0 & 0.2 \\
\hline Fibre cement & 0.8 & 0.2 & 0.15 & 0.02 & 0.9 & 0.1 \\
\hline Smooth metal & 0.8 & 0.2 & 0.9 & 0.07 & 0.5 & 0.1 \\
\hline
\end{tabular}

hard to predict. The ICRP advise that a reference level of residual dose is set at the end of the emergency phase to help plan and optimise the strategy for recovery and that it should account for "social and economic factors" (ICRP, 2009). Therefore, a robust prediction of the long-term residual doses underpins decision-making in urban scenarios from transition.

The ERMIN model was developed to predict residual doses in urban areas and has been implemented in both RODOS and ARGOS decision support systems. ERMIN uses empirical parameters and functions to describe initial deposition and retention of radionuclides on urban surfaces including; roofs, walls, paved, grass, soil, trees, plants and interiors. With a library of idealised environments, it converts surface contamination to dose-rate and, with assumptions about the time spent indoors and outdoors in different environments, it estimates normal-living residual doses with and without clean-up options. ERMIN also estimates other consequences of clean-up including costs, exposure of workers and activity and amount of waste.

Under WP4 an investigation into the sources of uncertainty for ERMIN was undertaken to guide use of the current version, to make recommendations for future versions and to develop a wider range of case specific parametric sets to reduce prognostic uncertainty.

Distributions representing parameter uncertainty were compiled for initial deposition and subsequent retention on urban surfaces and for occupancy. For example, ERMIN has a single roof type with a single set of parameters describing deposition. Table 1 gives the default parameters that represent initial deposition of caesium in a soluble aerosol form, and it gives the material specific parameters compiled under WP4 from experiments and studies in the literature.

To investigate the impact of parameter uncertainty on the prediction of normal living residual dose, ERMIN was run using a Monte Carlo approach in which the compiled distributions were sampled independently to build up ensembles of predictions from which probabilistic inferences could be drawn. Figure 1a shows an example of a sensitivity analysis performed on the initial deposition to roof parameter under wet deposition conditions. ERMIN was repeatedly run for each material type and for the ERMIN default roof, and in each run values for initial deposition parameters were randomly drawn from normal distribution with the parameters in Table 1. All with all other ERMIN parameters kept at the default values. Similarly, Figure $1 \mathrm{~b}$ shows the sensitivity of the occupancy parameter (the fraction of time a person spent indoors), in the multi-storey environment.

The Monte Carlo approach was used to generate uncertain dose projections for scenarios presented to some of the National Stakeholder Panels described in Montero et al. (2020), in addition a demonstration scenario was developed for the CONFIDENCE final dissemination workshop (Montero et al., 2019). In this scenario, a town with a mix of different environment types (brick house, lightweight houses and multistorey buildings) was assumed to be contaminated. Projections of residual dose with uncertainty were generated by repeatedly running ERMIN, and in each run independently sampling the proposed distributions of initial deposition, retention, and occupancy parameters, and an arbitrary distribution intended to represent some of the variation in urban environment type of the chosen settlement. Figure 1c shows the uncertainty on the predicted residual dose without any clean-up strategies applied.

Predictions of residual doses in urban scenarios are just one input into a decision-making process that will involve many different stakeholders with different priorities and concerns which will reflect not only how they are impacted but how they expect to be impacted. For the demonstration scenario, several different clean-up strategies were suggested; do nothing, a high waste strategy comprising recovery actions expected to be effective at dose reduction but that also generate waste and a low waste strategy comprising recovery actions that produce less waste but which may not be so effective. Figure $1 \mathrm{~d}$ shows the predicted uncertainty on the duration that annual doses remain above a $5 \mathrm{mSv} \mathrm{y}^{-1}$ reference level, when nothing is done and when low waste and high waste strategies are applied and the uncertainties on impact when given as a duration are striking.

\section{Agricultural scenarios}

According to the ICRP, as mentioned above, the residual dose is the reference level set to optimise protective measures. The prediction of the residual dose is therefore the final aim in agricultural scenarios. However, due to the large number of components that constitute them this prediction is subject to uncertainty.

The approach to the agricultural scenarios in the transition phase may be addressed knowing the objectives to achieve, the topics to be addressed and the criteria for decision to be used. 


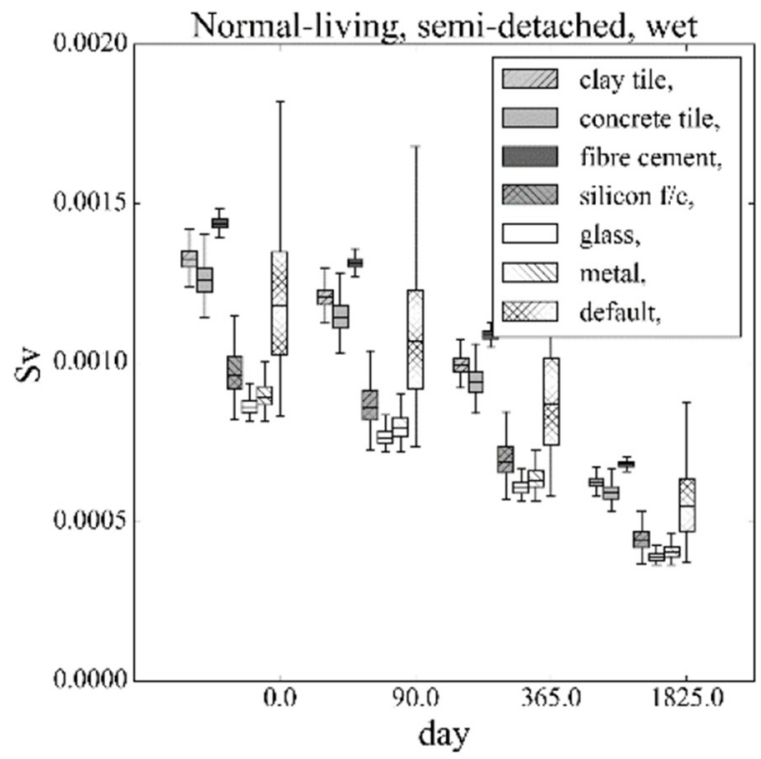

a)

Predicted residual external dose

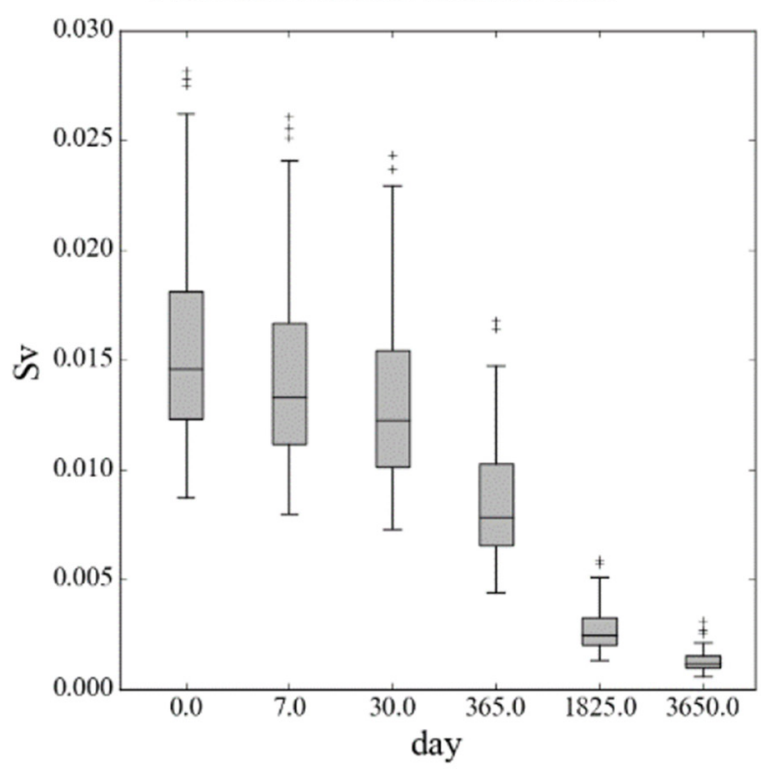

c)

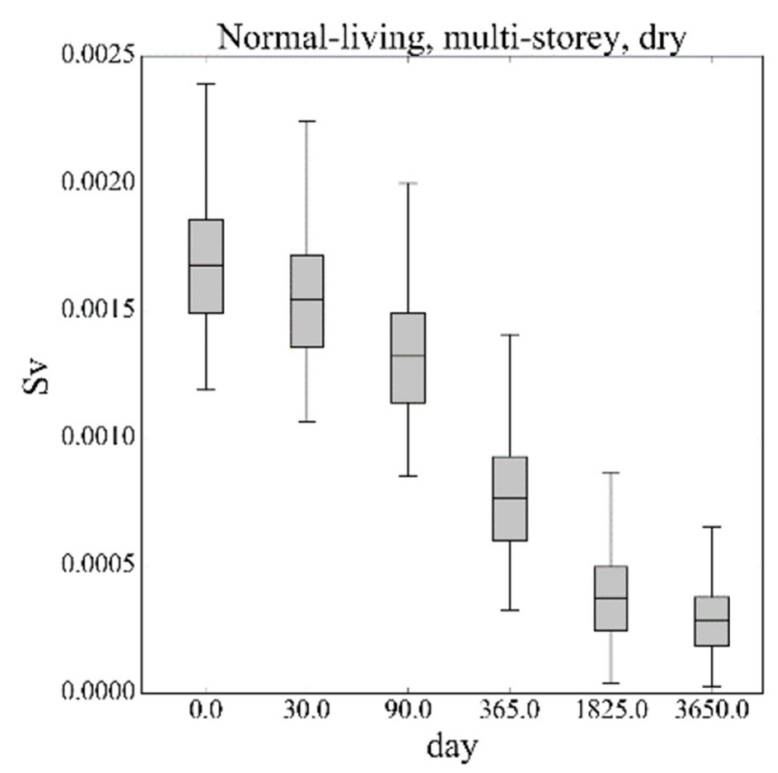

b)

Duration that dose exceeds reference level $0.005 \mathrm{~Sv}$

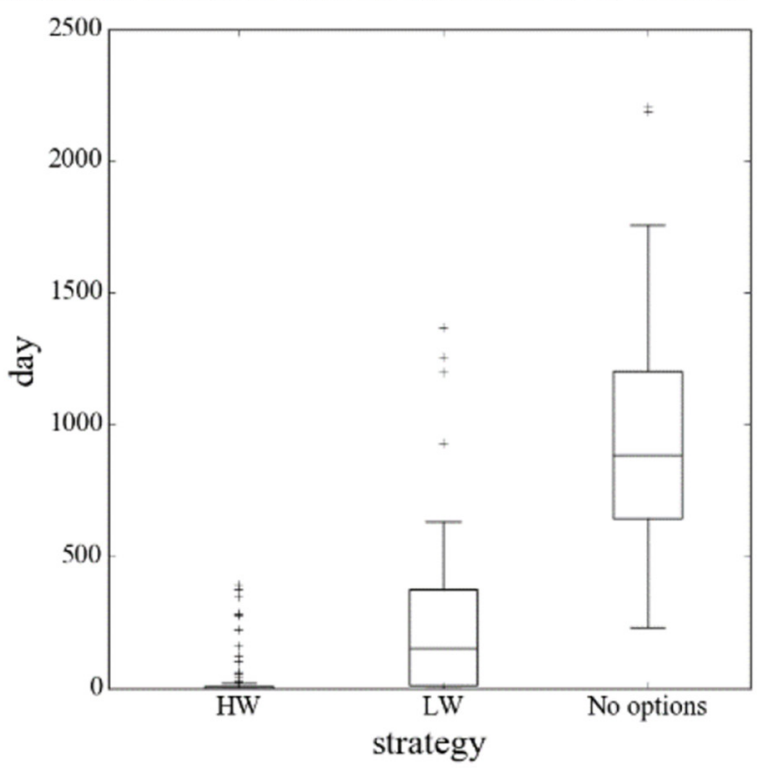

d)

Fig. 1. Examples of sensitivity and uncertainty analysis performed with ERMIN: (a) sensitivity to roof material of the predicted dose in a semidetached brick house environment following wet deposition; (b) sensitivity to occupancy of predicted dose in a multi-storey building environment following dry deposition; (c) uncertainty of predicted dose in an example scenario with no options applied; (d) uncertainty on duration that the residual annual dose will exceed $5 \mathrm{mSv}$ in an example scenario where three different strategies might be applied. All doses given are external normal-living integrated over 1 year from the time given from $1 \mathrm{MBqm}^{-2}$ of ${ }^{137} \mathrm{Cs}$ calculated by ERMIN. The box for each marker indicates the inter-quartile range (IQR), the range from quartile $1(\mathrm{Q} 1)$ to quartile $3(\mathrm{Q} 3), 50 \%$ of results are contained in the box, the line in the box is the 2nd quartile or the median. The lower whisker is Q1-1.5IQR and the upper whisker is Q3 + 1.5IQR. If none of the data are at or beyond the limit of the whisker then the whisker will be truncated. If the distribution is normal then nearly all results would lie within the range of the whiskers. The points beyond the whiskers are individual outlying results.

These allow stakeholders, experts and decision makers to identify and to evaluate different alternatives, and to deal proactively with complexity and judgment in decisionmaking. The participants' involvement will depend on both the objectives, topics and criteria of the situation, and on the type of participant; who may be directly involved, or can contribute to the solution or unaffected but interested. 


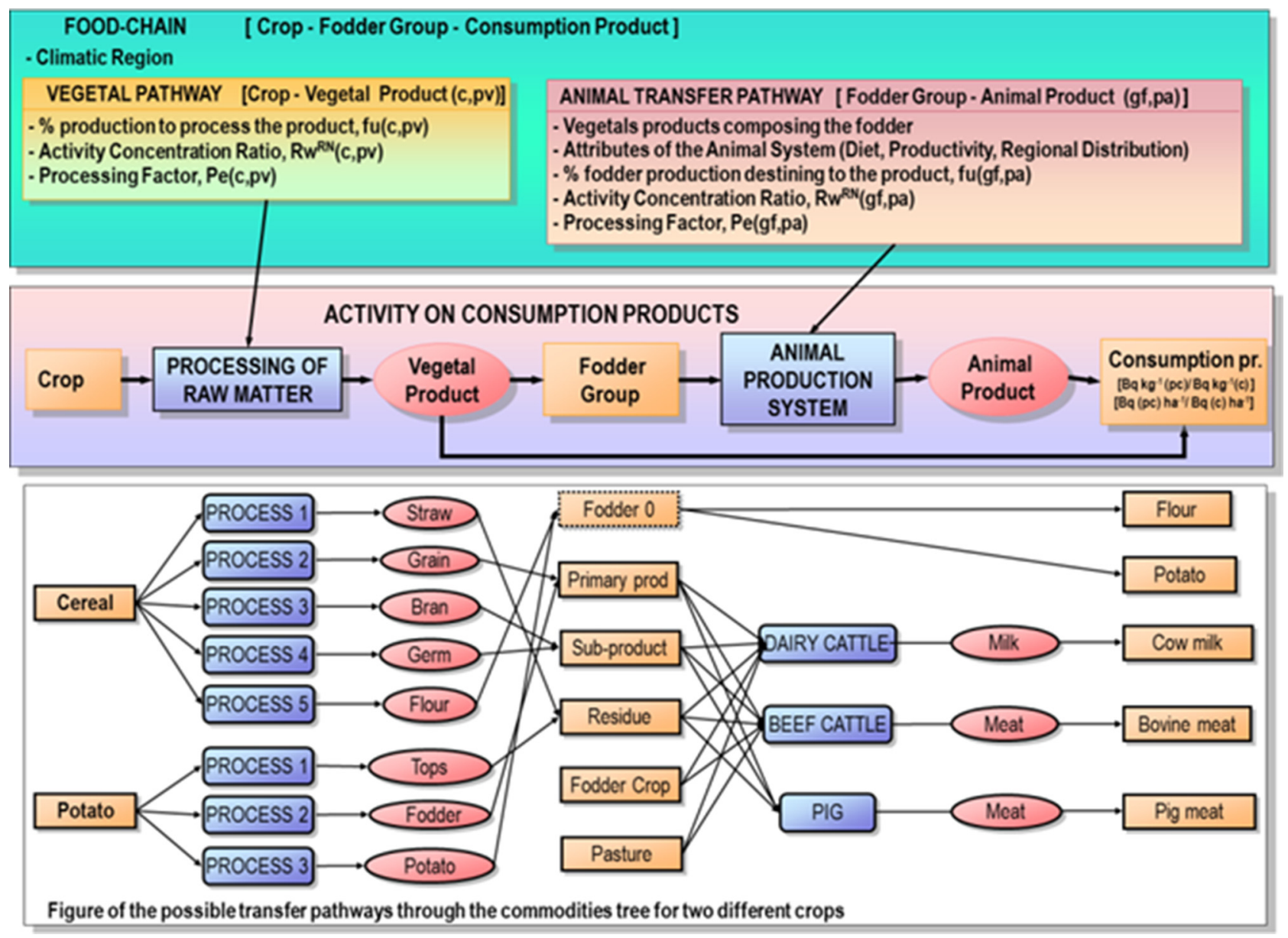

Fig. 2. Example of food transfer pathways for cereals and potatoes, considering elemental units.

All these issues can be identified in a decision-oriented scenario-analysis. These are narrative descriptions of potential futures that focus the attention on relationships between events and decisions that need to be taken. Following this premise, the main aspects in the construction process of agricultural scenarios are:

- the characterization of the different elements or elemental units as function of the parameters or attributes that influence the behaviour and transfer of radionuclides in them. Figure 2, shows an example of transfer pathway considering elemental units for two crop types:

- primary component: soil-plant/crop,

- secondary components: transfer pathway along food chain (Crop-animal-product),

- final component: affected population (inhabitants and consumers);

- the definition and characterization of the different action alternatives to be used in each one of these components;

- models and methods to estimate and measure the consequences (spatial-temporal evolution of the contamination without and with recovery actions);
- identification of other factors that could affect the practicability and optimisation of the strategies (social, economic, political, environmental and ethical values).

The scenario construction process in the transition phase follows a number of steps. The first one is the characterization of the radiological situation using an adequate monitoring programme, to know the real level of deposition on the affected area. Equally important is the environmental characterization of elemental units, in terms of parameters and attributes that affect and influence the behaviour of the deposited radionuclides. This structure facilitates not only the analysis of the fate of the radionuclides in the different components by means of assessment models, also the response of the elemental units to the different applicable recovery actions in terms of reducing external, inhalation or ingestion doses, activity concentrations or crop uptake.

These evaluation models are necessary to assess the spacetime evolution of the scenario with and without recovery actions, helping to define the objectives and to quantify the criteria for decision-making. Regarding the recovery actions, and depending on the contaminated scenario and the objectives to achieve, they can be applied individually on a determined 
elemental unit or several of them integrated in a joint action, not necessarily applied over the same elemental unit or in the same period of time. The decision-making process with the involvement of the stakeholders will develop, in each case, the best protection strategy designed to address the objectives defined previously.

This process, as a whole, has associated a series of uncertainties related to:

- the radiological situation of the scenario, contributing to the overall uncertainty associated with the estimated impact. The uncertainties are specifically related to the space-time evolution of the contamination and the prediction of the radiological situation in the long term, the results of the monitoring programme and the possible changes in the future land use of the scenario;

- the goals and criteria used in the design of the protection strategy, in particular, in the objectives pursued, the radiological criteria selected and other indicators such as the area and no. of persons affected;

- the protection strategy regarding; the effectiveness of recovery actions, the possible side-effects, the wastes generated and their disposal, the costs of the overall strategy;

- the social pressure regarding; the trust and confidence of the public in the actions to be carried out, the acceptability of the protection strategy, possible conflicting interests among the affected population and/or affected economic activities within the affected area.

Finally, the involvement of stakeholders in decisionmaking is also subject to uncertainties, in particular, "how to learn from the stakeholders and the public, their preferences on clean-up and recovery strategies and integrate them into decision-making, recognising that they may be unclear on their valuation of these" (French et al., 2020).

Regarding the radiological situation, certain components of the scenario, in particular soils, may accumulate large amounts of radionuclides depending on the chemical form of the radionuclide and soil properties. For $\mathrm{Cs}$, $\mathrm{Sr}$ and I isotopes, there is now a good understanding of the underlying environmental processes that can lead to exposure.

Once deposited, the behaviour of these radionuclides in soils is mainly governed by physico-chemical processes that determine their fixation, mobility and bioavailability (Trueba et al., 2015). Table 2 shows the processes and parameters that affect the behaviour of radionuclides in soils. However, the parameters governing these processes can be affected by changes in the activity concentrations and fluxes of radionuclides along the food chain, giving rise to uncertainties in the predictions of the individual doses.

Furthermore, agricultural systems are complex and not homogeneous. Even within a particular region or area, there are multiple variables to be considered, which are inherently related to the affected systems, such as: climate, soil type and its properties, type of crops, seasonality, agricultural practices, etc.

It is important to highlight that the uncertainties may be enhanced as a function of the assessment models used to determine the activity concentrations and doses, for instance the Terrestrial Food Chain and Dose Module (FDMT)
Table 2. Soil processes and parameters associated to the behaviour of radionuclides in soils

\begin{tabular}{ll}
\hline Soil processes and parameters & $\begin{array}{l}\text { Soil capacity affecting } \\
\text { radionuclide behaviour }\end{array}$ \\
\hline $\begin{array}{l}\text { Infiltration process } \\
\text { Texture }\end{array}$ & Infiltration capacity \\
Structure & \\
Clay content, organic matter content & \\
Cation exchange capacity & \\
Vertical migration process & Water holding capacity \\
Texture & \\
Bulk density & \\
Organic matter content & \\
Permeability & \\
Sorption/desorption process & Physico-chemical \\
Clay content, organic matter content & retention capacity \\
Cation exchange capacity & \\
pH content & \\
Root uptake process & ${ }^{137}$ Cs transfer capacity \\
Exchangeable potassium content & ${ }^{90} \mathrm{Sr}$ transfer capacity \\
Exchangeable calcium content & \\
\hline
\end{tabular}

(Müller et al., 2003; Gering et al., 2010) included in the JRODOS (Ievdin et al., 2010; Landman et al., 2016) and ARGOS Decision Support Systems and SYMBIOSE. These are not only due to the calculation models themselves, but also to the parameters used. Models usually have default parameters, specific for a certain climatic region that may not be appropriate for other climatic regions in Europe (Thørring et al., 2016).

Region-specific parameters imply a more realistic assessment, affecting the reduction of the uncertainties of the predicted doses and the response of the potential recovery strategies to be applied. In this framework a $\mathrm{PhD}$ is being carried out to study, rank and map the influence of regional factors on the radiological risk due to the food chain exposure pathway (García-Puerta et al., 2017). Figure 3 schematically shows the methodological approach followed for Spanish soils.

Regarding the objectives and criteria used in the design of a recovery strategy, a literature review of the radiological criteria applicable in emergency situations has been accomplished to analyse how the implementation of a strategy could have consequences on the transition and long-term phases (Montero et al., 2018). These consequences are very difficult to predict and so represent sources of uncertainties themselves.

\section{Discussion}

The objective of WP4 of CONFIDENCE was to improve the preparedness and response in the transition, by identifying and trying to reduce uncertainties in the subsequent planning of management of the long-term exposure situation. The first task was to identify and investigate sources of uncertainty and so 


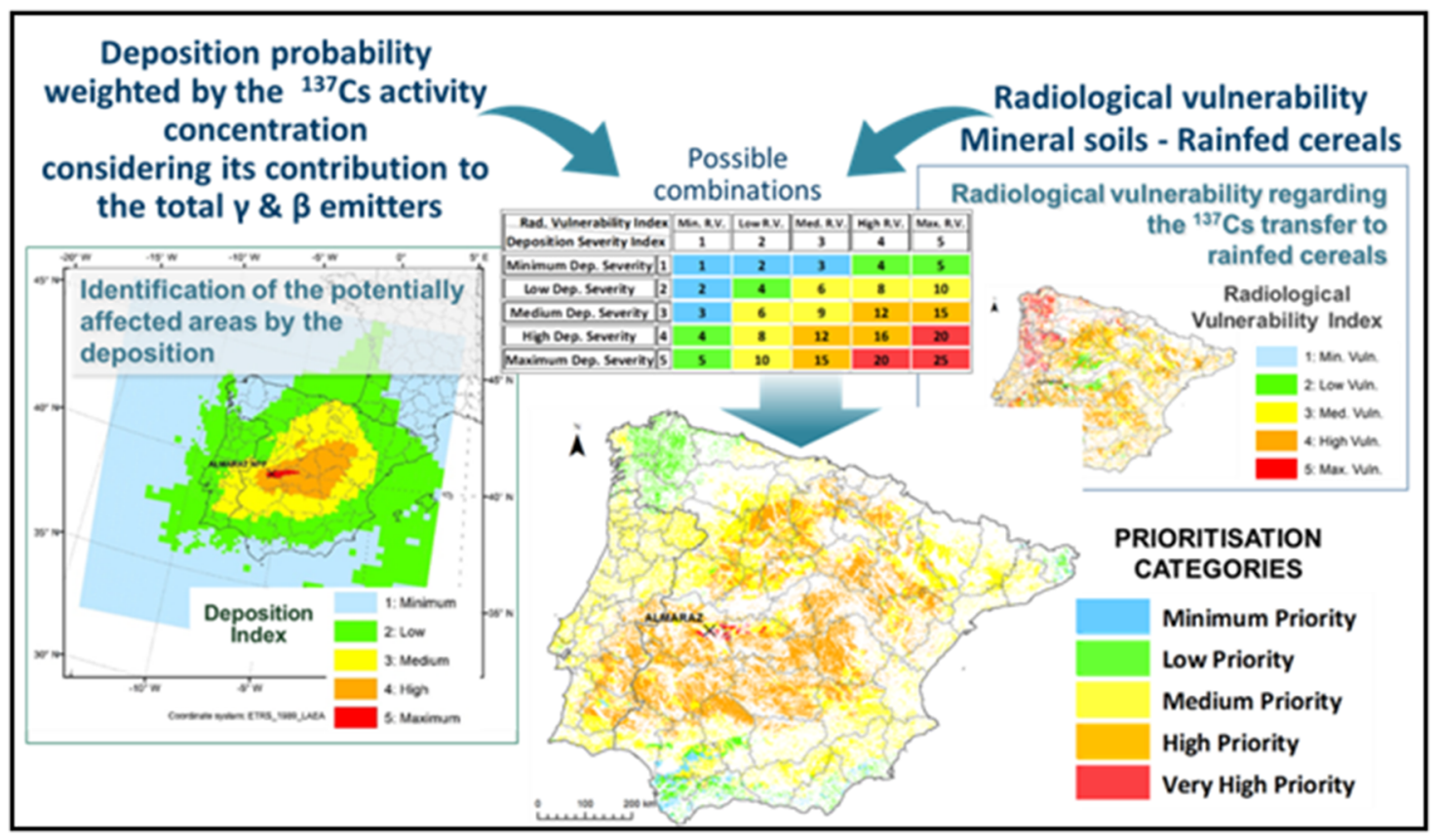

Fig. 3. Methodological approach to study the influence of regional factors on the radiological risk due to the food chain exposure pathway.

frame the scene at the point of transition regarding uncertainty of what might be described as "technical" information, for example the degree of contamination, the predicted residual dose or the effectiveness of recovery options, etc. as well as identify "non-technical" sources of uncertainty. It was then the purpose of subsequent tasks in the work package to investigate how stakeholders process all the information and uncertainties, both technical and non-technical (Durand et al., 2020; Montero et al., 2020).

Urban and agricultural scenarios were investigated separately and this is justified since radiological processes, pathways of exposure and the recovery options that may be deployed are generally different. But it is important to recognise that a real event will have elements of both and indeed the concept of residual dose explicitly includes all pathways.

The urban scenario investigation focussed on uncertainty in residual dose predictions, especially the component from external exposure to radionuclides deposited in an urban environment. Because it is predictions of residual dose along with a reference dose that delineate the area and duration of impact and so define who is considered to be a stakeholder at transition, and reduction of residual doses is usually one objective of recovery strategies. Therefore, residual dose prediction underpins all recovery decisions. However, it is important to stress that, for stakeholders, residual dose may not be the most important consideration and that other recovery objectives (for example maintaining social cohesion and economic renewal) may be higher priorities than reduction.

The urban scenario investigation looked at how sources of uncertainty could impact on the residual dose prediction uncertainty and at ways that uncertainty could be reduced. Charnock (2018, Appendix 2) gives recommendations for both the use of the ERMIN model in its current form and for its development to better handle uncertainty in future versions.

The agricultural scenario investigation focussed on the uncertainty related to the behaviour and fate of radionuclides in the soil-plant system and their transfer along the food chain pathway. The prediction of food contamination and ingestion doses to humans is a key element in the implementation and management of recovery actions.

The prediction relies on the radiological assessment of the affected scenario, the ability of the modelling to predict the time dependence of the transfer process, with and without recovery actions and the availability of reliable parameters. Regionspecific parameters imply a more realistic assessment, affecting the reduction of the uncertainties of the estimated doses and the response of the potential recovery strategies to be applied.

One observation that arises from both investigations is that handling and evaluating "technical" uncertainty in decision support or modelling systems is only useful if that uncertainty can then be communicated to stakeholders in a way that is meaningful and useful. This is a discussion beyond just the transition and recovery phase, but involves the whole incident management and is the primary focus of WP6 of CONFIDENCE.

Acknowledgement. The work described in this paper was conducted within the CONFIDENCE project which was part of the CONCERT project. The CONCERT project received funding from the Euratom Research and Training Programme (2014-2018) under grant agreement No. 662287.

Disclaimer (Art. 29.5 GA). This publication reflects only the author's view. Responsibility for the information and views expressed therein lies entirely with the authors. The European Commission is not responsible for any use that may be made of the information it contains. 


\section{References}

Charnock TW, Andersson KG, Montero M, Trueba C. 2018. Addressing the uncertainties in urban/inhabited scenarios. CONCERT Deliverable D9.20. Available from https://www. concert-h2020.eu/en/Publications.

Durand V, Maître M, Crouaïl P, Schneider T, Sala R, Marques-Nunes P, Paiva I, Monteiro Gil O, Reis M, Hilliard C, Tafili V, Twenhöfel C, Van Asselt E, Trueba C, Montero M, Duranova T. 2020. Towards an improved decision-making process to better cope with uncertainties following a nuclear accident. Radioprotection 55 (HS1). https://doi.org/10.1051/radiopro/2020023.

French S, Haywood S, Oughton D, Turcanu C. 2020. Different types of uncertainty. Radioprotection 55(HS1). https://doi.org/10.1051/ radiopro/2020029.

García-Puerta B, Trueba C, Montero M. 2017. Local factors to make risk maps, for potential nuclear accident affected areas, to be applied in the decision-making process. In: Proceedings of the 4th International Conference on Radioecology and Environmental Radioactivity, 4th-8th September 2017, Berlin (Germany).

Gering F, Raskob W, Charnock T. 2010. New model for agricultural countermeasures in RODOS and ARGOS. Radioprotection 45(5): 63-S76.

ICRP. 2009. Application of the Commission's recommendations to the protection of people living in long-term contaminated areas after a nuclear accident or a radiation emergency. ICRP Publication 111. Ann. ICRP 39(3).

Ievdin I, Trybushnyi D, Zheleznyak M, Raskob W. 2010. RODOS reengineering: Aims and implementation details. Radioprotection 45(5 Supplément): S181-S189.

Landman C, Raskob W, Trybushnyi D, Ievdin I. 2016. Creating and running worldwide accident scenarios with JRodos. Radioprotection 51(HS1): S27-S30.
Montero M, Trueba C, García-Puerta B, Sala R, Andresz S, Schneider T, Maître M, Croüail P, Durand V, Charron S. 2018. Addressing the uncertainties in agricultural scenarios. CONCERT Deliverable D9.21. Available from https://www.concert-h2020.eu/en/Publica tions.

Montero M, Trueba C, Sala R, Nunes P, Reis M, Paiva I, GarcíaPuerta B, Charnock T. 2019. Block 4: Strategies involving stakeholders in the transition phase. Scenario based facilitated discussion. Introduction to the scenario. In: CONFIDENCE Final Dissemination Workshop. Coping with uncertainties for improved modelling and decision making in nuclear emergencies, 2-5 December 2019, Bratislava, Slovakia.

Montero M, Sala R, Trueba C, García-Puerta B, Abelshausen B, Bohunova J, Croüail P, Durand V, Duranova T, Hilliard C, Maitre M, Mitrakos D, Monteiro Gil O, Nunes P, Paiva I, Reis M, Schneider T, Skuterud L, Smith V, Tafili V, Thørring H, Turcanu C, Twenhöfel C, Van Asselt E. 2020. Stakeholder involvement through national panels and surveys to address the issues and uncertainties arising in the preparedness and management of the transition phase. Radioprotection 55(HS1). https://doi.org/ 10.1051/radiopro/2020022.

Müller H, Gering F, Pröhl G. 2003. Model description of the terrestrial food chain and dose module. FDMT in RODOS PV6.0. RODOS(RA3)-TN(03)06. V1.1.

Thørring H, Dyve JE, Lahtinen J, Montero M, Simon-Cornu M, Trueba C. 2016. Human food chain modelling. Summary of results from the FDMT and SYMBIOSE model runs. COMET Milestone (IRA-Human-M3).

Trueba C, Montero M, García-Puerta B. 2015. Soil vulnerability maps for predictive purposes in decision making processes for postaccident recovery in Spain. In: Second NERIS Workshop Proceedings, May 2015, Milano, Italy.

Cite this article as: Charnock T, Andersson K, Trueba C, Montero M. 2020. Uncertainties confronting stakeholders and decision-makers in planning intervention in urban and agricultural scenarios in the transition phase of a radiological emergency. Radioprotection 55(HS1): S119-S125 